\title{
Optimal Scaling of Multicommodity Flows in Wireless Ad Hoc Networks: Beyond The Gupta-Kumar Barrier
}

\author{
Shirish Karande ${ }^{\dagger}$, Zheng Wang $^{\dagger}$, Hamid R. Sadjadpour ${ }^{\dagger}$, J.J. Garcia-Luna-Aceves ${ }^{\ddagger}$ \\ Department of Electrical Engineering ${ }^{\dagger}$ and Computer Engineering ${ }^{\ddagger}$ \\ University of California, Santa Cruz, 1156 High Street, Santa Cruz, CA 95064, USA \\ $\ddagger$ Palo Alto Research Center (PARC), 3333 Coyote Hill Road, Palo Alto, CA 94304, USA \\ Email:\{karandes,wzgold, hamid, jj\}@ soe.ucsc.edu
}

\begin{abstract}
We establish a tight max-flow min-cut theorem for multi-commodity routing in random geometric graphs. We show that, as the number of nodes in the network $n$ tends to infinity, the maximum concurrent flow (MCF) and the minimum cut-capacity scale as $\Theta\left(n^{2} r^{3}(n) / k\right)$ for a random choice of $k \geq \Theta(n)$ sourcedestination pairs, where $r(n)$ is the communication range in the network. We exploit the fact that the MCF in a random geometric graph equals the capacity of an ad-hoc network under the protocol model and interference-free communication to derive scaling laws for interference-constrained network capacity. We generalize all existing results reported to date by showing that the per-commodity capacity of the network scales as $\Theta(1 / r(n) k)$ for the single-packet reception model suggested by Gupta and Kumar, and as $\Theta(n r(n) / k)$ for the multiple-packet reception model suggested by others. More importantly, we show that, if the nodes in the network are capable of multiple-packet transmission and reception, then it is feasible to achieve the optimal scaling of $\Theta\left(n^{2} r^{3}(n) / k\right)$, despite the presence of interference. This result provides an improvement of $\Theta\left(n r^{2}(n)\right)$ over the highest achieved capacity reported to date. In stark contrast to the conventional wisdom that has evolved from the Gupta-Kumar results, our results show that the capacity of ad-hoc networks can actually increase with $n$ while the communication range tends to zero!
\end{abstract}

\section{INTRODUCTION}

The price, performance and form factors of sensors, processors, storage elements, and radios today are at a point that the age of viable large-scale ad-hoc networks would appear to be finally upon us. Alas, Gupta and Kumar's [1] seminal work cautions us to the contrary. They studied the capacity of wireless ad-hoc networks with $n$ nodes when receivers are static, transmit or receive one packet at a time, and the network traffic consists of $n$ unicast sessions. Their conclusion was a rather negative one: The capacity of ad-hoc networks does not scale with an increase in network size.

Gupta and Kumar's analysis [1] applies to the traditional view of ad hoc networking in which protocols are based on a one-to-one communication paradigm aimed at avoiding multiple access interference (MAI). However, a number of recent advances in cooperative communication and generalizations of routing are challenging the long-held view that avoiding interefrence is the way to maximize throughput in ad hoc networks. For example, network coding (NC) [2] generalizes routing by permitting processing of packets at intermediate nodes. In certain network configurations (refer to illustrations in [3], [4]) some nodes can utilize NC to concurrently transmit multiple packets. Many-to-one and many-to-many communication is also feasible under a variety of other cooperative techniques [5]-[7].

Co-operative protocols that provide performance benefits in specific network configurations need not scale well with the network size. In particular, Liu et al. [8] proved another disheartening result: NC cannot increase the throughput order of wireless ad-hoc networks for multi-pair unicast applications under half-duplex communication. However, in a recent challenge, Garcia-Luna-Acves et al. [6] call for the realization of ad hoc networks that scale by embracing MAI through the use of multi-packet reception (MPR) at the receivers. They show that, if the nodes in the network are capable of MPR, then the order capacity of a network with $n$ unicast sessions grows as $\Theta(r(n))$, where $r(n)$ is the communication range. This represents a gain of $\Theta\left(n r^{2}(n)\right)$ over the throughput order of $\Theta(1 / n r(n))$ reported by Gupta and Kumar.

Interestingly, the prior work on the capacity of wireless networks, which we summarize in Section II, has focused on what is attainable with specific approaches to handle MAI. No prior work has focused on first establishing what is the optimal capacity of a wireless network in the absence of MAI, and then determining whether that capacity is attainable when MAI is present. This is precisely the focus and overall contribution of this paper.

Section III presents the first contribution of this paper. We model a random network with $n$ nodes, a homogeneous communication range of $r(n)$, and unicast traffic for $k$ sourcedestination (S-D) pairs. In the absence of interference, such a network orresponds to a random geometric graph with an edge between any two nodes separated by a distance less than $r(n)$. We define a combinatorial interference model based on random geometric graphs, and use it to express all the protocol models used in the past and a model that we later use to show that the optimal capcity of a wireless network is indeed attainable. We introduce a protocol model in which nodes have the ability to decode correctly multiple packets transmitted concurrently from different nodes, and transmit concurrently multiple packets to different nodes. We refer to this as the multi-packet transmission and reception (MPTR) protocol model. 
Section IV presents the second contribution of this paper, which is the characterization of the optimal interference-free capacity of a wireless network. The task of concurrently maximizing the data-rate for $k \mathrm{~S}$-D pairs is an instance of the multi-commodity flow problem. Hence, the maximum concurrent multi-commodity flow-rate (MCF) in a random geometric graph equals the interference free capacity (i.e., the optimal capacity) of the network. To derive upper bounds on the optimal network capacity, we use the fact that the MCF is less than the minimum capacity of a multi-commodity cut for any arbitrary graph. The max-flow min-cut theorem by Ford and Fulkerson [9] establishes that this bound is tight for a single commodity. However, in general, the min-cut does not provide a tight bound on the max-flow [10]. The bound is known to be tight only for special cases, such as planar graphs [11], and in general exhibits a gap of at least $\Theta(\log k)$ [12]. We establish a tight max-flow min-cut theorem for random geometric graphs for the first time, and show that $\Theta\left(n^{2} r^{3}(n) / k\right)$ is a tight bound on the optimal capacity of a wireless network.

Section V presents our third contribution, which consists of generalizing prior results by Gupta and Kumar and by GarciaLuna-Aceves et al., and proving that the optimal capacity of wireless networks is attainable in the presence of MAI. We utilize the max-flow min-cut theorem of Section IV to deduce tight order bounds for the capacity of random networks under various interference models. We show that the percommodity capacity, under the protocol model suggested in [1], exhibits a tight order bound of $\Theta(1 / r(n) k)$. This result generalizes Gupta and Kumar's result to any $k \geq \Theta(n)$ SD pairs. Similarly, we generalize Garcia-Luna-Aceves et al.'s analysis for the MPR protocol model. We show that, under the MPR model, the per-commodity capacity of the network scales as $\Theta(n r(n) / k)$, which means that it is bounded away from the optimal capacity by a factor of $\Theta\left(n r^{2}(n)\right)$. Furthermore, the analysis in [6] implicitly assumes the existence of a tight maxflow min-cut theorem for random geometric graphs; therefore, the results in this paper fill an important gap in their analysis.

We show that MPTR achieves the optimal capacity of $\Theta\left(n^{2} r^{3}(n) / k\right)$. Hence, MPTR provides a gain of $\Theta\left(n r^{2}(n)\right)$ over MPR and any previously reported feasible capacity. What is just as stricking is that MPTR can achieve the dual objective of increasing capacity and decreasing the transmission range as $n$ increases. This is in stark contrast to the commonly held view that the capacity of multihop wireless networks cannot increase as the number of nodes increases. Indeed, our results demonstrate that the capacity of ad-hoc networks can actually increase with $n$ while the communication range tends to zero! Section VI addresses the impact of our results on the design of protocols for future wireless ad hoc networks.

\section{RELATED WORK}

There have been many contributions on the capacity study of wireless ad hoc networks and span unicast, multicast and broadcast traffic. Due to space limitations, however, we only mention a few of them that focus on unicasting.

A number of papers have extended the results by Gupta and Kumar [1], which showed a gap between the upper and lower bounds on capacity under the physical model. Franceschetti et al. [13] closed this gap using percolation theory.

Several techniques aimed at improving the capacity of wireless ad hoc networks have been analyzed. Grossglauser and Tse [14] demonstrated that a non-vanishing capacity can be attained at the price of long delivery latencies by taking advantage of long-term storage in mobile nodes. Someworks demonstrated that changing physical layer assumptions such as using multiple channels [15] or MIMO cooperation [16] can change the capacity of wireless networks.

Ozgur et al. [17] proposed a hierarchical cooperation technique based on virtual MIMO to achieve linear capacity. They showed that the optimal per-session capacity of an ad-hoc network is bounded as $O(n \log n)$, and a constant per-session capacity of $\Theta(1)$ is achievable. Our work is significantly different from this work, in terms of the the model and assumptions used to derive the results. Ozgur et. al. consider the information-theoretic model, and assume that the network employs heterogenous hop-sizes, at times requiring a direct communication between widely separated nodes. In contrast, our work is based on the protocol model and assumes a homogenous transmission range, which is a more realistic assumption.

Cooperation can be extended to the simultaneous transmission and reception at the various nodes in the network, which can result in significant capacity improvement [5]. As we have stated, Garcia-Luna-Aceves et al. [6] showed that using MPR at the receivers can increase the order capacity of wireless networks subject to unicast traffic.

A generalization of the max-flow min-cut theorem to multiple commodities is not feasible in arbitrary graphs. However, the seminal paper by Leighton and Rao [10] showed that the gap between the max-flow and min-cut is at most $\Theta(\log n)$. In a recent work, Madan et. al. [18] have utilized the Leighton and Rao's work to derive bounds for the capacity of ad-hoc networks. They focus on the special case where $k=\Theta\left(n^{2}\right)$ and the single packet reception (SPR) model.

Our work is inspired by the analysis of Leighton and Rao. Because we are able to deduce a tight max-flow min-cut theorem, our bounds are tighter than those reported by Madan et al. [18]. Furthermore, our work is applicable to a wider variety of protocol models and traffic patterns.

\section{PRELIMINARIES}

\section{A. Network Model}

For a continuous region $R$, we use $|R|$ to denote its area. We denote the cardinality of a set $S$ by $|S|$, and by $\|x-y\|$ the distance between nodes $x$ and $y$. Whenever convenient, we utilize the indicator function $1_{\{P\}}$, which is equal to one if $P$ is true and zero if $P$ is false. $\operatorname{Pr}(E)$ represents the probability of event $E$. We say that an event $E$ occurs with high probability (w.h.p.) as $n \rightarrow \infty$ if $\operatorname{Pr}(E)>(1-(1 / n))$. We employ the standard order notations $O, \Omega$, and $\Theta$.

We assume a random wireless network with $n$ nodes distributed uniformly in a unit-square. In our model, as $n$ goes to infinity, the density of the network also goes to infinity. Therefore, our analysis is applicable to dense networks. Furthermore, we assume a fixed transmission range $r(n)$ for all 
the nodes in the network. Thus, the network topology can be characterized using a random geometric graph, which we denote by $G_{r}$ and define next.

Definition 3.1: Random Geometric Graph $G_{r}$ :

We associate a directed graph $G_{r}\left(V_{r}, E_{r}\right)$ with a wireless network formed by distributing $n$ nodes uniformly in a unit square. We represent the node-set by $V=\{1, \cdots, n\}$. Let the locations of these nodes be given by $\left\{X_{1}, \cdots, X_{n}\right\}$, the edge-set is then $E=\left\{(i, j) \mid\left\|X_{i}-X_{j}\right\| \leq r(n)\right\}$.

While the results in this paper can be extended to undirected graphs, it is more convenient for us to use directed graphs because we use edge-coloring techniques in our work. In the case of undirected graphs, the argument should be based on vertices (receivers) rather than edges. Also note that, in this paper, we permit two edges for a pair of connected vertices with possibly different capacity in each direction.

We assume that the network operates using a slotted channel and, in the absence of interference, the data rate in each time slot for every transmitter-receiver pair is a constant of value $\mathrm{W}$ bits/slot. Given that $\mathrm{W}$ does not change the order capacity, we normalize its value to 1 . Hence, we say that the interferencefree capacity of each edge in $G_{r}$ is equal to 1 .

Gupta and Kumar [1] have proved the following criteria for the connectivity of $G_{r}$.

Lemma 3.2: For a random distribution of $n$ nodes in a unitsquare, the graph $G_{r}$ is connected with high probability as $n \rightarrow \infty$, iff. $r(n) \geq r_{c}(n)=\Theta(\sqrt{\log n / n})$.

In a dense network, interference is the primary constraint on the capacity of the network. Like Madan et al. [18], we describe the interference of a network by the following generic model.

Definition 3.3: Combinatorial Interference Model:

The interference model for the graph ${ }^{1} G(V, E)$ is determined by a function $I: E \rightarrow P(E)$, where $P(E)$ is the power set of $E$, i.e., the set of all possible subsets of $E$. For every $e \in E$, $I(e)$ represents an interference set such that, a transmission on edge $e$ is successful if and only if (iff) there are no concurrent transmissions on any $\hat{e} \in I(e)$. An interference model can be restricted to a sub-graph $H\left(V_{H}, E_{H}\right)$ by defining a function $I_{H}: E_{H} \rightarrow P\left(E_{H}\right)$ such that $I_{H}(e)=I(e) \bigcap E_{H}$.

The various protocol models that have been proposed in the past can now be expressed as special cases on $G_{r}$.

Gupta and Kumar [1] studied a single packet reception (SPR) protocol model under which a transmission from node $i$ to receiver $j$ is successful iff $\left\|X_{i}-X_{j}\right\| \leq r(n)$ and if $\left\|X_{j}-X_{k}\right\| \geq(1+\eta) r(n)$ for any other transmitter $k$. Here $\eta$ is a guard-zone that is assumed to be constant for the entire network. Moreover, all the nodes operate in half-duplex mode. The following definition expresses this model in terms of the notation we have introduced.

Definition 3.4: Single-Packet Reception (SPR) Model:

Let $e=\left(e^{+}, e^{-}\right) \in E$, then the interference set for edge $e$ is defined as

$$
\begin{aligned}
I_{\mathrm{SPR}}(e) & =J(e)-e \\
J(e) & =\left\{\hat{e} \in E_{r} \mid\left\|X_{\hat{e}^{+}}-X_{e^{-}}\right\| \leq(1+\eta) r(n)\right\}
\end{aligned}
$$

${ }^{1}$ Note that $G_{r}$ denotes random geometric graph while $G$ represents general graph.
Garcia-Luna-Aceves et al. [6] generalized the above model to account for MPR capability at the receivers. According to their MPR protocol model a node $i$ can simultaneously receive all the packets transmitted by nodes within a distance $r(n)$ iff there are no transmitters at a distance greater than $r(n)$ but less than $(1+\eta) r(n)$. Furthermore, if a node $j$ transmits a packet to node $i$, then it cannot simultaneously transmit a packet to any other node in the network.

\section{Definition 3.5: Multi-Packet Reception (MPR) Model:}

$$
\begin{aligned}
I_{\mathrm{MPR}}(e) & =I_{\mathrm{SPR}}(e)-(A(e)-B(e)) \forall e \in E \text { where } \\
A(e) & =\left\{\hat{e} \in E_{r} \mid\left\|X_{\hat{e}^{+}}-X_{e^{-}}\right\| \leq r(n)\right\} \\
B(e) & =\left\{\hat{e} \in E_{r} \mid \hat{e}^{+}=e^{+}\right\}
\end{aligned}
$$

We also consider the case in which nodes have MPR and MPT capabilities, i.e., can decode multiple concurrent transmissions and can transmit concurrently multiple packets to different nodes. We capture the MPR and MPT capabilities with a simple yet representative, multi-packet transmission and reception (MPTR) protocol model, which is defined below.

Definition 3.6: Multi-Packet Transmission and Reception (MPTR) Model:

$$
I_{\mathrm{MPTR}}(e)=I_{\mathrm{MPR}}(e)-B(e) \quad \forall e \in E
$$

It is important to highlight some of the features of the above model. The MPTR protocol model still restricts the nodes to operate in a half-duplex mode. Moreover, this model is identical to the MPR protocol model in terms of the nodes that are permitted to transmit within the vicinity of a receiver $i$, i.e., both models prohibit transmission from a node $j$ such that $r(n)<\left\|X_{i}-X_{j}\right\| \leq(1+\eta) r(n)$. Thus, the difference between the MPR and MPTR protocol models stems purely from the fact that, under the MPTR model, a node $j$ transmitting a packet to node $i$ can simultaneously transmit packets to other nodes in the network.

We assume that the traffic in the network is generated by unicast communication between $k$ source-destination (S-D) pairs. We associate a rate vector $\lambda=\left[\lambda_{1}, \cdots, \lambda_{k}\right]$ with these $k$ pairs. We assume the data rate for each S-D pair to be nonzero. Hence, without loss of generality (w.l.g) the rate vector can be written as $\lambda=\left[f D_{i}, \cdots, f D_{k}\right]$ where $f \in \mathbb{R}_{+}$and $D_{i} \in[1 / 2,1]$ for $1 \leq i \leq k$. We refer to the parameter $f$ as the concurrent flow rate and to $D=\left[D_{1}, \cdots, D_{k}\right]$ as the demand vector.

Definition 3.7: Feasible Flow Rate:

Given $k$ S-D pairs $\{(s(1), d(1)), \ldots,(s(k), d(k))\}$, a rate vector $\lambda=\left[f D_{i}, \cdots, f D_{k}\right]$ is feasible if there exists a spatial and temporal scheme for scheduling transmissions such that by operating the network in a multi-hop fashion, and buffering at intermediate nodes when awaiting transmission, every source $s(i)$ can send $\lambda_{i}$ bits/sec on average to the chosen destination $d(i)$. A flow rate $f$ is feasible for a demand vector $D=\left[D_{1}, \cdots, D_{k}\right]$ iff $\lambda=\left[f D_{i}, \cdots, f D_{k}\right]$ is a feasible rate vector.

Definition 3.8: Capacity of Random Networks: The capacity per commodity of a network is $\Theta(f(n))$ if under 
a random placement of $n$ nodes, a random choice of $k$ S-D pairs and for an arbitrary demand vector we have:

$$
\begin{aligned}
\lim _{n \rightarrow \infty} \operatorname{Pr}(c f(n) \text { is feasible flow rate }) & =1 \\
\liminf _{n \rightarrow \infty} \operatorname{Pr}\left(c^{\prime} f(n) \text { is infeasible flow rate }\right) & <1
\end{aligned}
$$

for some $c>0$ and $c<c^{\prime}<+\infty$.

In the following sections we repeatedly utilize the well known Chernoff bounds:

Lemma 3.9: Chernoff Bounds: Consider $N$ i.i.d random variables $Y_{i} \in\{0,1\}$ with $p=\operatorname{Pr}\left(Y_{i}=1\right)$. Let $Y=\sum_{i=1}^{N} Y_{i}$. Then for every $c>0$ there exist $0<\delta_{1}<1$ and $\delta_{2}>0$ such that

$$
\begin{gathered}
\operatorname{Pr}\left(Y \leq\left(1-\delta_{1}\right) N p\right)<e^{-c N p} \\
\operatorname{Pr}\left(Y \geq\left(1+\delta_{2}\right) n p\right)<e^{-c N p}
\end{gathered}
$$

\section{B. Graph Theory Results}

We review some defintions from graph theory. In particular, note that the task of identifying a feasible flow rate can be posed as a multi-commodity flow problem, specifically the kcommodity flow problem.

Definition 3.10: $k$-Commodity Flow Problem:

Consider a directed graph $G(V, E)$ with a capacity function $c: E \rightarrow[0,1]$. Let $\{(s(1), d(1)), \ldots,(s(k), d(k)))$ be $k \mathrm{~S}-\mathrm{D}$ pairs, with a demand vector $D \in[1 / 2,1]^{k}$. Let $f \in \mathbb{R}_{+}$be a concurrent flow rate. Find flow functions $f_{i}: E \rightarrow \mathbb{R}_{+}$for $1 \leq i \leq k$, which satisfy the following flow constraints:

Capacity Constraint : $\forall e \in E$

$$
\sum_{1 \leq i \leq k} f_{i}(e) \leq c(e)
$$

Flow Conservation : $\forall v \neq s(i), d(i)$

$$
\sum_{e: e^{+}=v} f_{i}(e)=\sum_{e: e^{-}=v} f_{i}(e)
$$

Demand Satisfaction : $1 \leq i \leq k$

$$
\sum_{e: e^{+}=s(i)} f_{i}(e)=\sum_{e: e^{-}=d(i)} f_{i}(e)=f D_{i}
$$

Flow functions that satisfy the above constraints are called feasible. Other inputs to the problem being fixed, a flow rate $f$ is said to feasible iff the above problem has a solution. Furthermore, let $f^{*}$ be the MCF such that the above problem has a feasible solution. A wireless network can be represented by an equivalent graph with capacity functions determined by the interference. Thus, the MCF in an equivalent graph can be perceived as the maximum flow that can be routed in a network. Additionally, if w.h.p. $f^{*}$ is the MCF for any graph formed by a random distribution of nodes, sources and destinations, then the capacity of the wireless network is also $f^{*}$.

Consider the following additional definitions.

Definition 3.11: Vertex Cut:

Given a node set $V$, a cut is the separation of the vertex set $V$ into two disjoint and exhaustive sets $\left(S, S^{C}\right)$. We shall often reference a cut just by the set $S$.
Definition 3.12: Multi-commodity Cut Capacity:

Given a graph $G(V, E)$, a capacity function $c: E \rightarrow[0,1]$ and a cut $\left(S, S^{C}\right)$. The multi-commodity cut capacity is defined as

$$
\Upsilon_{G, S}=\frac{\sum_{e \in E} 1_{\left[e^{+} \in S, e^{-} \in S^{C}\right]} c(e)}{\sum_{i: s(i) \in S, d(i) \in S^{C}} D_{i}}
$$

Definition 3.13: Minimum Cut Capacity:

Given a graph $G(V, E)$, a capacity function $c: E \rightarrow[0,1]$ and a cut $\left(S, S^{C}\right)$. The minimum multi-commodity cut capacity is defined as

$$
\Upsilon_{G}=\min _{S \subset V} \frac{\sum_{e \in E} 1_{\left[e^{+} \in S, e^{-} \in S^{C}\right]} c(e)}{\sum_{i: s(i) \in S, d(i) \in S^{C}} D_{i}}
$$

It is well-known that the minimum cut-capacity provides an upper bound on the maximum flow rate.

Lemma 3.14: For any $k$-commodity flow $f^{*} \leq \Upsilon_{G}$

\section{OPtimal CAPACiTy}

We show that for random geometric graphs, the MCF provides a tight approximation of the minimum-cut capacity. This relationship implies a tight characterization of the interferencefree capacity of wireless ad-hoc networks with a homogenous transmission range. Our approach can be summarized as follows: For a particular demand vector, we provide an upper bound by showing that there exists a multi-commodity cut in $G_{r}$ of order $O(g(n))$ and a lower bound by constructing a flow of order $\Omega(g(n))$ in a sub-graph $H_{r} \subseteq G_{r}$. These results along with the following Lemmas prove that the capacity of $H_{r}$ and $G_{r}$ has a tight bound $\Theta(g(n))$.

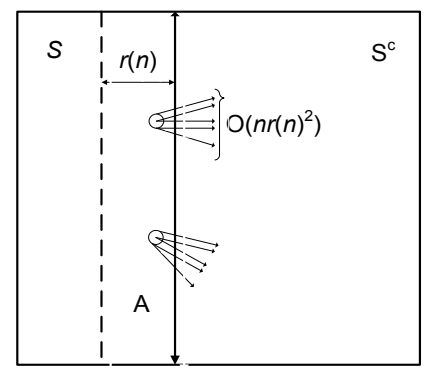

Fig. 1. A bi-partitioning of the unit square

Lemma 4.1: A graph $G(V, E)$ and a sub-graph $H\left(V_{H}, E_{H}\right)$ satisfy the following two properties: (a) If $f$ is a feasible flowrate in $H$ then $f$ is feasible in $G$; and (b) the capacity of a cut $\left(S, S^{C}\right)$ in $G$ is always greater than or equal to the capacity of the cut in $H$.

Proof: To prove Part (a) of the lemma, let $f_{H, i}$ for $1 \leq i \leq k$ be the flow functions associated with the feasible flow of rate $f$ in $H$. Note that these flow functions satisfy the constraints in Definition 3.10. We construct a flow in $G$ of rate $f$ with the following flow functions: For $1 \leq i \leq k$ let $f_{G, i}: E \rightarrow \mathbb{R}_{+}$such that

$$
f_{G, i}(e)=\left\{\begin{aligned}
f_{H, i}(e) & \text { if } e \in E_{H} \\
0 & \text { otherwise }
\end{aligned}\right.
$$

Now, if we show that the functions $f_{H, i}$ satisfy the flow constraints, then the flow rate $f$ is feasible in $G$. Definition 
3.10 states that $c(e) \geq 0$ for all the edges. As a result, $\forall e \in$ $E-E_{H}$ the capacity constraints are satisfied trivially, given that $f_{G, i}(e)=0$ for such edges. Furthermore, $\forall e \in E_{H}$ we have

$$
\sum_{1 \leq i \leq k} f_{H, i}(e)=\sum_{1 \leq i \leq k} f_{G, i}(e) \leq c(e)
$$

Therefore, $f_{G, i}$ satisfy the capacity constraint. In addition, note that the following equations hold $\forall v \in V$ :

$$
\begin{aligned}
\sum_{e \in E: e^{-}=v} f_{G, i}(e) & =\sum_{e \in E_{H}: e^{-}=v} f_{G, i}(e)+\sum_{e \in E-E_{H}: e^{-}=v} f_{G, i}(e) \\
& =\sum_{e \in E_{H}: e^{-}=v} f_{H, i}(e)+0 \\
\sum_{e \in E: e^{+}=v} f_{G, i}(e)= & \sum_{e \in E_{H}: e^{+}=v} f_{G, i}(e)+\sum_{e \in E-E_{H}: e^{+}=v} f_{G, i}(e) \\
& =\sum_{e \in E_{H}: e^{+}=v} f_{H, i}(e)+0
\end{aligned}
$$

Eqs. (16) and (17) imply that the net in-flow and the net out-flow, under $f_{G, i}$ and $f_{H, i}$, is identical for all nodes and commodities. Therefore, $f_{G, i}$ satisfies the flow conservation and demand constraints.

To show Part (b), observe that

$$
\Upsilon_{G, S}=\Upsilon_{H, S}+\frac{\sum_{e \in E-E_{H}} 1_{\left[e^{+} \in S, e^{-} \in S^{C}\right]} c(e)}{\sum_{i: s(i) \in S, d(i) \in S^{C}} D_{i}}
$$

Because $c(e) \geq 0$ for all edges, we have $\Upsilon_{G, S} \geq \Upsilon_{H, S}$.

\section{A. Upper Bound}

We utilize the following properties of $G_{r}$.

Lemma 4.2: If $r(n) \geq r_{c}(n)$ then w.h.p. graph $G_{r}$ is such that: (a) The minimum vertex degree $\nabla \geq \Theta\left(n r^{2}(n)\right)$, and (b) the maximum vertex degree $\Delta \leq \Theta\left(n r^{2}(n)\right)$.

Proof: We first show that $\nabla \geq \Theta\left(n r^{2}(n)\right)$. A node $v$ in $G_{r}$ is connected to all the nodes in a disk of radius $r(n)$ centered at $v$. The area of this disk is $\pi r^{2}(n)$. Given a uniformly random distribution of nodes, the probability of another node $u$ lying within this disk is $\pi r^{2}(n)$. Consider a random variable $Y_{v, u} \in\{0,1\}$ which is equal to one iff node $u$ is connected to node $v$. The degree of node $v$ can be written as $\operatorname{deg}(v)=\sum_{u \in V-\{v\}} Y_{v, u}$. Therefore, the Chernoff Bound (11) implies that $\forall c>0$ there exists a $0 \leq \delta \leq 1$ such that

$$
\operatorname{Pr}\left(\operatorname{deg}(v) \leq(1-\delta) n \pi r^{2}(n)\right)<e^{-c n \pi r^{2}(n)}
$$

From the union bound we obtain

$\operatorname{Pr}\left(\nabla \leq(1-\delta) n \pi r^{2}(n)\right)<n \operatorname{Pr}\left(\operatorname{deg}(v) \leq(1-\delta) n \pi r^{2}(n)\right)$

Given that $r(n) \geq r_{c}(n)$ we have $r(n) \geq c_{1} \sqrt{\log n / n}$ for some $c_{1}>0$. Therefore, Eqs. (19) and (20) imply that

$$
\operatorname{Pr}\left(\nabla \leq(1-\delta) \pi n r^{2}(n)\right)<n e^{-c \pi c_{1} \log n}=1 / n^{c \pi c_{1}-1}
$$

Now, Lemma 3.9 tell us that we can choose $\left.c \geq 2 /\left(\pi c_{1}\right)\right)$ and corresponding $0<\delta_{1}<1$ such that

$$
\operatorname{Pr}\left(\nabla \geq(1-\delta) \pi n r^{2}(n)\right)>1-(1 / n)
$$

We use similar arguments to show that $\Delta \leq \Theta\left(n r^{2}(n)\right)$ w.h.p.. This fact follows from Eq. (8), which implies that $\forall c>0$ there exists a $0 \leq \delta \leq 1$ such that

$$
\operatorname{Pr}\left(\operatorname{deg}(v) \geq\left(1+\delta_{1}\right) n \pi r^{2}(n)\right)<e^{-c n \pi r^{2}(n)}
$$

The union bound and the fact that $r(n) \geq c_{2} \sqrt{\log n / n}$ implies that

$$
\operatorname{Pr}\left(\Delta \geq(1+\delta) \pi n r^{2}(n)\right)<n e^{-c \pi c_{2} \log n}
$$

Therefore, there exists a $\delta_{2}>0$ s.t

$$
\operatorname{Pr}\left(\Delta \leq\left(1+\delta_{2}\right) \pi n r^{2}(n)\right)>1-(1 / n)
$$

Consider the cut $S$ described by Figure 1 . The cut consists of all the nodes in the rectangular region of a constant area.

Lemma 4.3: If the network consists of $k \geq \Theta(\log n)$ S-D pairs then w.h.p. a region $R$ of constant area $|R|$ contains $\Theta(k)$ sources with destinations outside region $R$.

Proof: Let $Y_{i} \in 0,1$ be a random variable that is equal to one iff the ith S-D pair is such that $s(i)$ belongs to region $R$ and $d(i)$ does not. Under a uniformly random placement of nodes

$$
\operatorname{Pr}\left(Y_{i}=1\right)=|R|(1-|R|)
$$

The total number of S-D pairs satisfying the required condition can be represented by $Y=\sum_{1}^{k} Y_{i}$. If $k \geq c \log n$ the Chernoff Bounds imply the existence of constants $c_{1}=1 /(c|R|(1-$ $|R|)), 0 \leq \delta_{1} \leq 1$ and $\delta_{2}>0$ s.t.

$$
\begin{aligned}
\operatorname{Pr}(Y \geq & \left.\left(1-\delta_{1}\right)|R|(1-|R|) k\right)>1-e^{-c_{1} k|R|(1-|R|)} \\
& \geq 1-e^{-c_{1} c|R|(1-|R|) \log n}=1-(1 / n) \\
\operatorname{Pr}(Y \leq & \left.\left(1+\delta_{2}\right)|R|(1-|R|) k\right)>1-e^{-c_{1} k|R|(1-|R|)} \\
& =1-e^{-c_{1} c|R|(1-|R|) \log n}=1-(1 / n)
\end{aligned}
$$

Furthermore, consider the subset $A$ in $S$ defined by a strip of dimension $1 \times r(n)$. The total number of vertices in $\mathrm{A}$ is $\Theta(n r(n))$ because of the uniform distribution of nodes in the network.

Theorem 4.4: If $r(n) \geq r_{c}(n)$ and $k \geq \Theta(\log n)$, then the capacity of the cut $S$ is $\Upsilon_{G_{r, S}}=O\left(n^{2} r^{3}(n) / k\right)$ w.h.p.

Proof: According to the definition of $G_{r}$ two nodes are connected iff they are separated by a distance less than $r(n)$. Consequently, if an edge cuts across $S$ then it has to be incident upon a node at a distance less than $r(n)$ from the boundary separating $S$ and $S^{C}$, i.e. the head of the edge should lie in the subset $A$ of dimension $r(n) \times 1$. Furthermore, for each node in $A$ the maximum number of edges cutting across the cut is bounded by $\Delta$

$$
\sum_{e \in E} 1_{\left[e^{+} \in S, e^{-} \in S^{C}\right]} \leq|A| \Delta
$$


In the absence of interference $c(e)=1$ for all the edges. Hence,

$$
\begin{gathered}
\Upsilon_{G_{r}, S}=\frac{\sum_{e \in E_{r}} 1_{\left[e^{+} \in S, e^{-} \in S^{C}\right]}}{\sum_{i: s(i) \in S, d(i) \in S^{C}} D_{i}} \\
\leq \frac{|A| \Delta}{\sum_{i: s(i) \in S, d(i) \in S^{C}} D_{i}}
\end{gathered}
$$

According to Lemma 4.3, there exists $c_{1}>0$ s.t. the total number S-D pairs across the cut is $c_{1} k$. Furthermore, by Definition 3.10 the demand for each pair is at least $1 / 2$. Hence,

$$
\Upsilon_{G_{r}, S} \leq \frac{2|A| \Delta}{c_{1} k}
$$

Lemma 4.2 implies that there exists a $c_{2}>0$ s.t. $\Delta<$ $c_{2} n r^{2}(n)$, while uniform distribution of nodes in the network implies that there exists a $c_{3}>0$ s.t. $|A| \leq c_{3} n r(n)$. Hence,

$$
\Upsilon_{G_{r}, S} \leq 2 c_{2} c_{3} n^{2} r^{3}(n) /\left(c_{1} k\right)
$$

Any cut in $G_{r}$ has a capacity greater than the minimum cut capacity $\Upsilon_{G}$. Consequently Theorem 4.5 implies the following Corollary.

Corollary 4.5: If $r(n) \geq r_{c}(n)$ and $k \geq \Theta(\log n)$ then $\Upsilon_{G_{r}}$ is upper bounded as $O\left(n^{2} r^{3}(n) / k\right)$.

\section{B. Lower Bound}

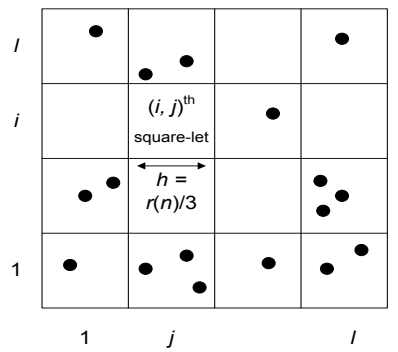

Fig. 2. Decomposition of network area into $l^{2}$ squarelets

To describe a capacity-achieving flow in a more generic setting, we use an important result from parallel and distributed computing. Consider a mesh of $l^{2}$ processing units with $l$ processors in each row and column. Let each processor be a source and destination of exactly $h$ packets. The problem of routing the $h l^{2}$ packets to their destinations is known as $h \times h$ permutation routing and can be characterized by the following result [19].

Lemma 4.6: If in a single slot, each processor can transmit one packet each to its immediate horizontal and vertical neighbors, then an $h \times h$ permutation routing in a $l \times l$ mesh can be performed deterministically in $h l / 2+o(h l)$ steps.

We utilize the following corollary that can be readily deduced from the above Lemma.

Corollary 4.7: If a processor is capable of transmitting at least $\eta$ packets to each of its neighbors in each slot, then an $h \times h$ permutation routing in a $l \times l$ mesh can be performed deterministically in $O(h l / \eta)$ steps.
Now consider a sub-graph $H_{r} \subseteq G_{r}$ obtained by employing location based constraints on the edge-set. In order to describe these constraints, we first define a location dependent hash function.

Definition 4.8: Index Function $\zeta$ :

Divide the network area into $l^{2}$ squarelets [20] of side-length $a=r(n) / 3$, as shown in Figure 2 . Let $\zeta$ be a function that associates an index $(i, j)$ with a squarelet in the $i_{t h}$ column and $j_{t h}$ row. Furthurmore, the index assigned to each squarelet is associated with each vertex in the squarelet.

We obtain $H_{r}$ by removing all edges, except those connecting two nodes in vertically or horizontally adjacent squarelets. We do not necessarily have to consider $H_{r}$ in order to obtain a lower bound on the interference-free capacity. However, the performance bounds for $H_{r}$ play an important role when we analyze interference constrained networks in Section V.

Definition 4.9: Geographically Restricted Sub-Graph $H_{r}$ : The graph $H_{r}\left(V_{r}, E_{r, H}\right)$ is a sub-graph of $G_{r}$ with an identical node-set and an edge-set defined as:

$$
E_{r, H}=\left\{e \in E \mid \zeta\left(e^{-}\right)=(a, b) \Rightarrow \zeta\left(e^{+}\right)=(a \pm 1, b \pm 1)\right\}
$$

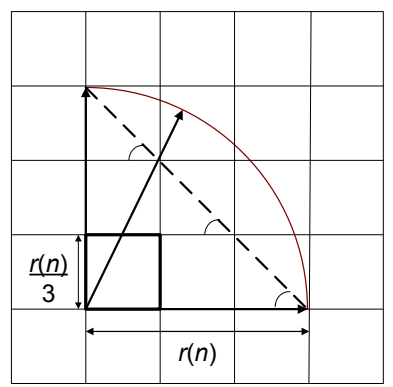

Fig. 3. A geometric proof to show that any two points in adjacent square-lets are within a distance $r(n)$ of each other. The proof follows from the fact that the chord of a circle lies within it.

Consider some of the properties of the squarelets and $H_{r}$.

Lemma 4.10: [20] If $r(n) \geq r_{c}(n)$ then w.h.p. the total number of nodes in any squarelet are $\Theta\left(n r^{2}(n)\right)$.

Proof: The area of a squarelet is equal to $\Theta\left(r^{2}(n)\right)$. Hence, the proof is identical to that of Lemma 4.2.

Lemma 4.11: If $r(n) \geq r_{c}(n)$ and $k \geq \Theta(n)$, then w.h.p. the total number of sources in any squarelet are $\Theta\left(k r^{2}(n)\right)$ and the total destinations in any squarelet are $\Theta\left(k r^{2}(n)\right)$.

Proof: For $1 \leq i \leq k$ let $Y_{i, m} \in 0,1$ be a random variable that is equal to one iff source $s(i)$ belongs to the $m^{t h}$ squarelet. Let $Y_{m}=\sum_{1}^{k} Y_{i, m}$ represent the total number of sources in the squarelet. Because $r(n) \geq r_{c}(n)$, Eq.(11) implies

$$
\left.\operatorname{Pr}\left(Y_{m} \leq(1-\delta) k r^{2}(n)\right)\right)<e^{-(c k \log n) / n}
$$

The total number of squarelets in a unit square is equal to

$$
(3 / r(n)) \times(3 / r(n)) \leq c_{1} n / \log n
$$

Therefore, the union bound implies that

$$
\begin{array}{ll}
\operatorname{Pr} & \text { (min. no. of nodes in a squarelet }<\Theta\left(k r^{2}(n)\right) \\
\leq & (\text { total no. of squarelets }) \times e^{-(c k \log n) / n} \\
\leq & \left(c_{1} n / \log n\right) \times e^{-(c k \log n) / n} \\
= & c_{1} /\left(n^{(c k / n)-1} \log n\right)
\end{array}
$$


Thus, $k \geq \Theta(n)$ guarantees the required convergence and hence we can say that each squarelet has at least $\Theta\left(k r^{2}(n)\right)$ sources. The upper bound on the number of sources and the bounds on the number of destinations can be calculated similarly.

Theorem 4.12: If $r(n) \geq r_{c}(n)$ and $k \geq \Theta(n)$, then w.h.p. the maximum flow rate $f_{H}^{*}$ in $H_{r}$ is at least $\Theta\left(n^{2} r^{3}(n) / k\right)$.

Proof: The proof follows from mapping various components of the above defined problem to the $h \times h$ permutation routing problem. Let us map each squarelet to a processor. Consequently, for the chosen size of squarelets, the network equates to a mesh of $l^{2}$ processors with $l=3 / r(n)$. Assume that each source intends to transmit $D_{i}$ as the $\mathrm{i}^{\text {th }}$ element of the demand vector. Because $D_{i} \leq 1$, Lemma 4.11 implies that the total number of bits to be transmitted to and from each squarelet are at most $h \leq c k r^{2}(n)$. Finally, note that any two nodes in adjacent squarelets are within a distance $r(n)$. Fig. 3 provides a geometric proof for this fact; an alternative proof can be easily obtained by employing the Pythagoras theorem. In each slot, we can send one packet along each edge between two adjacent squarelets. Therefore, Lemma 4.10 implies that

$$
\begin{aligned}
\eta & =(\text { min. no. of edges between adjacent squarelets }) \\
& \leq(\text { min. no. of nodes per squarelet })^{2} \leq c_{1} n^{2} r^{4}(n)
\end{aligned}
$$

Hence, the total number of slots $\gamma$ required to complete the desired routing is

$$
\begin{aligned}
\gamma & \leq\left(c_{2} h l / \eta\right) \\
& \leq c_{2} \times\left(c k r^{2}(n)\right) \times(3 / r(n)) \times\left(1 / c_{1} n^{2} r^{4}(n)\right) \\
& =\left(3 c_{2} c k / c_{1} n^{2} r^{3}(n)\right)
\end{aligned}
$$

We can repeat the above routing periodically to guarantee a flow rate of $f=(1 / \gamma) \geq \Theta\left(n^{2} r^{3}(n) / k\right)$. By definition, the max-flow rate is greater than any other feasible flow rate, and the theorem follows.

Aggregating the above results we have the following conclusion.

Theorem 4.13: If $r(n) \geq \Theta \sqrt{\log n / n}$ and $k \geq \Theta(n)$, then the max- flow $f_{G}^{*}$ in $G_{r}$ can be approximated tightly by the min-cut capacity $\Upsilon_{G_{r}}^{*}$ in $G_{r}$. Moreover, the $f_{G}^{*}$ and $\Upsilon_{G_{r}}^{*}$ scale as $\Theta\left(n^{2} r^{3}(n) / k\right)$.

Proof: Lemma 4.1 implies that $f_{G}^{*} \geq f_{H}^{*}$. Hence, the result follows from the lower bound provided by Theorem 4.12 and the upper bound provided by Corollary 4.5

Scaling laws for $k=\Theta(n)$ have been given special attention in the literature. Hence, it is worth stating explicitly the above results for this scenario.

Corollary 4.14: Consider an ad-hoc network described by a random placement of $n$ nodes in a unit square, with $\Theta(n)$ S-D pairs and a homogenous transmission range of $r(n) \geq$ $\Theta(\sqrt{\log n / n})$. The interference-free capacity of the network scales as $\Theta\left(n r^{3}(n)\right)$.

\section{INTERFERENCE-LIMITED CAPACITY}

\section{A. General Results on Interference Models}

Interference can severely limit the network capacity. In this section we obtain scaling laws for the SPR, MPR and MPTR interference models. We primarily focus on deducing lower bounds. Moreover, to facilitate a succinct analysis, we develop some additional terminology and establish some important results.

Recall from Section III that, for two different communication schemes $A$ and $B$ (e.g., SPR and MPR) defined in the same graph, their corresponding interference functions $I_{A}$ and $I_{B}$ are different.

Definition 5.1: Dual-Interference-Set:

Consider an edge set $E$ and an interference set $I(e)$ for an edge $e \in E$, as defined in Definition 3.3. The dual interference-set for $e$ is defined by $F(e)=\{\hat{e} \in E \mid e \in I(\hat{e})\}$, which is the set of edges that experience a collision on account of a transmission on edge $e$.

Definition 5.2: Dual Conflict Graph:

Given a graph $G(V, E)$ and an interference function $I$, we defined the dual conflict graph as $G_{D}\left(E, E_{D}\right)$, where $E_{D}=$ $\{(e, \hat{e}) \mid \hat{e} \in I(e))\}$.

Definition 5.3: Total Degree in Dual Conflict Graph: The total degree of each node in a dual conflict graph is equal to $|M(e)|$ where $M(e)=I(e) \bigcup F(e)$.

Similar to the work in [18], we have the following Lemma.

Lemma 5.4: Consider a graph $G(V, E)$ and interference $I$. Let $\kappa=\max _{e \in E}|M(e)|$. If $f$ is a feasible flow rate in the absence of interference, then flow rate $f_{I}=f /(1+\kappa)$ is feasible in presence of interference $I$.

Proof: In the absence of interference the capacity of each edge is assumed to be one. However, because of interference, all edges cannot be activated simultaneously. Let $\sigma$ be the minimum frequency with which each edge is activated without causing any interference conflicts. Then, for each edge we have $c(e) \geq 1 / \sigma$.

Observe that $\kappa$ is the maximum vertex degree of the dual conflict graph $G_{D}$. It is well known that, if $\kappa$ is the maximum vertex degree, then $\kappa+1$ colors are sufficient to provide a proper vertex coloring [21]. Thus, we can partition the edgeset $E$ into $1+\kappa$ subsets such that no two edges in the same subset interfere. Consequently, we can periodically activate these subsets to realize $c(e) \geq 1 /(1+\kappa)$ for each edge. Thus, a feasible flow rate $f_{I}=f /(1+\kappa)$ can be obtained by scaling the flow functions associated with $f$ by a factor of $1 /(1+\kappa)$.

The maximum vertex degree does not provide a tight bound on the minimum number of colors required to provide a proper vertex coloring. Hence, in order to analyze a wider variety of protocol models, we introduce the concept of interference clones.

Definition 5.5: Interference Clone:

Two edges $e_{1}, e_{2}$ are said to be interference-clones under function $I$ if they satisfy the conditions that $M\left(e_{1}\right)=M\left(e_{2}\right)$.

Lemma 5.6: Clone Piggy-backing Lemma: Consider a graph $G(V, E)$ along with interference functions $I_{A}$ and $I_{B}$, then $I_{A}$ and $I_{B}$ are such that:

1) $\kappa=\max _{e \in E}\left|M_{A}(e)\right|$

2) $\forall e \in E$ there exists a set $M_{A, \bar{B}}(e) \subseteq M_{A}(e)$ such that every edge belonging $M_{A, \bar{B}(e)}$ is an interference-clone of $e$ under $I_{B}$. Further, let $\mu=\min _{e \in E}\left|M_{A \bar{B}}(e)\right|$. 
If $f$ is a feasible flow rate in $G$ without any interference, $f_{I_{A}}=f /(1+\kappa)$ is a feasible flow rate in $G$ under the $I_{A}$ interference function and $\kappa$ as its correponding parameter, then $f_{I_{B}}=f(1+\mu) /(1+\kappa)$ is feasible in presence of interference defined by $I_{B}$.

Proof: Consider the interference defined by $I_{A}$. From Lemma 5.4 we know that there exists a conflict free periodic schedule which can activate each edge at least once every $(1+\kappa)$ slots. Let us represent this schedule by an indicator function $\alpha(e, \tau)$ which equals one iff edge $e$ is active in slot $\tau$. Note that the capacity of each edge under schedule $\alpha$ is given by

$$
c_{\alpha}(e)=\Sigma_{\tau} \alpha(e, \tau)=1 /(1+\kappa) .
$$

Now let us use this schedule in the presence of interference $I_{B}$. Observe that, for every $e, \alpha$ allocates a distinct slot for each edge in $M_{A, \bar{B}}(e) \bigcap\{e\}$. Consequently, every edge has $\left|M_{A, \bar{B}}(e)\right|$ interference clones scheduled in slots distinct from each other and the edge itself. In addition, note that if an edge is activated in a time slot meant for one of its interference clones, then it does not lead to any conflict. Therefore, we can define a new conflict-free schedule $\beta$ such that $\beta(e, \tau)=1$ iff there exits an $e_{1} \in M_{A, \bar{B}}(e) \bigcap\{e\}$ such that $\alpha\left(e_{1}, \tau\right)=1$. Given that $\mu=\min _{e \in E}\left|M_{A \bar{B}}(e)\right|$, the capacity of each edge under schedule $\beta$ for interference $I_{B}$, is given by

$$
\begin{gathered}
c_{\beta}(e)=\Sigma_{e_{1} \in M_{A, \hat{B}}} \Sigma_{\tau} \alpha\left(e_{1}, \tau\right) \\
\leq(1+\mu) \times(1 /(1+\kappa))
\end{gathered}
$$

Accordingly, a feasible flow of $f_{I_{B}}=f(1+\mu) /(1+\kappa)$ can be obtained by scaling all the flow functions associated with the inference-free flow by a factor of $(1+\mu) /(1+\kappa)$.

In the subsequent discussion, we find it convenient to deduce a bound for a particular interference model and then show that it applies to a wider set of models. In order to facilitate such arguments, we define the following partial order.

Definition 5.7: Partial Order of Interference Models:

An interference function $I_{A}$ is said to be more restrictive than $I_{B}$, represented as $I_{A} \preceq I_{B}$, iff every edge satisfies the conditions that $I_{B}(e) \subseteq I_{A}(e)$.

Lemma 5.8: Consider a graph $G(V, E)$ along with interference $I_{A}$ and $I_{B}$. If $I_{A} \preceq I_{B}$, then a feasible flow rate under $I_{A}$ remains feasible under $I_{B}$.

Proof: A conflict free schedule under $I_{A}$ remains conflict free under $I_{B}$. Hence we can say that

$$
c_{A}(e) \leq c_{B}(e)
$$

where $c_{A}(e)$ and $c_{B}(e)$ represent the edge capacities under each interference model. Therefore, if a particular flow satisfies the capacity constraints under $I_{A}$, it necessarily satisfies those same constraints under $I_{B}$.

\section{B. Lower Bounds}

Direct analysis of the interference models can be tedious. Hence, for mathematical convenience, we consider the following restrictive models. Lemma 5.8 allows us to utilize the performance limits under these restrictive models to indirectly bind the capacity under the interference models for SPR, MPR and MPTR.

We define a restrictive interference model that introduces more restrictions (i.e., collisions) on the interference set for each edge than those strictly dictated by the original interference model. We will show that, under this restrictive model, the order of the lower bound capacity achieves the upper bound under the original (non-restrictive) interference model.

Definition 5.9: Restricted SPR (RSPR) Model:

$$
\begin{aligned}
I_{\mathrm{RSPR}}(e) & =W(e)-\{e\} \quad \forall e \in E_{r, H} \\
\text { where } W(e) & =\bigcup_{\hat{e}: \zeta\left(\hat{e}^{-}\right)=\zeta\left(e^{-}\right)} M_{S P R}(\hat{e})
\end{aligned}
$$

Definition 5.10: Restricted MPR (RMPR) Model:

$$
\begin{aligned}
I_{\mathrm{RMPR}}(e) & =W(e)-U(e) \forall e \in E_{r, H} \\
\text { where } U(e) & =\left\{\hat{e} \in E_{r, H} \mid \hat{e}^{-}=e^{-}\right\}
\end{aligned}
$$

Definition 5.11: Restricted MPTR (RMPTR) Model:

$$
\begin{aligned}
I_{\mathrm{RMPTR}}(e) & =W(e)-V(e) \forall e \in E_{r, H} \\
\text { where } V(e) & =\bigcup_{\hat{e}: \zeta\left(\hat{e}^{-}\right)=\zeta\left(e^{-}\right)} U(\hat{e})
\end{aligned}
$$

Consider the following properties of the restricted models.

Lemma 5.12: For the graph $H_{r}$, we have a partial order defined by (i) $I_{\mathrm{SPR}} \preceq I_{\mathrm{MPR}} \preceq I_{\mathrm{MPTR}}$, (ii) $I_{\mathrm{RSPR}} \preceq I_{\mathrm{RMPR}} \preceq$ $I_{\mathrm{RMPTR}}$, (iii) $I_{\mathrm{SPR}} \preceq I_{\mathrm{RSPR}}$ (iv) $I_{\mathrm{RMPR}} \preceq I_{\mathrm{MPR}}$ (v) $I_{\mathrm{RMPTR}} \preceq$ $I_{\mathrm{MPTR}}$

Proof: (Sketch) The proof for the partial orders (i) to (iii) follows directly from the definitions. Partial order (iv) follows from the fact that $J(e) \subseteq W(e)$ and $U(e) \subseteq A(e)-B(e)$. Similarly, the fact that $A(e) \subseteq V(e)$ implies partial order (v).

Lemma 5.13: If $r(n) \geq r_{c}(n)$, then all edges $e \in E_{r, H}$ have $|M(e)|=\Theta\left(n^{2} r^{4}(n)\right)$ under interference described by either of the models: $I_{\mathrm{SPR}}, I_{\mathrm{MPR}}, I_{\mathrm{MPTR}}, I_{\mathrm{RSPR}}, I_{\mathrm{RMPR}}, I_{\mathrm{RMPTR}}$.

Proof: From Lemma 5.12 we can conclude that RSPR is the most restrictive model, while MPTR is the least restrictive model. Further note that $F_{\mathrm{RSPR}}(e) \subseteq W(e)$ and $I_{\mathrm{MPTR}}(e) \subseteq$ $M_{\mathrm{MPTR}}(e)$. Hence, it suffices to prove the following:

$$
\begin{array}{r}
\gamma_{\max }=\max _{e \in E_{r, H}}|W(e)|=O\left(n^{2} r^{4}(n)\right) \\
\gamma_{\min }=\min _{e \in E_{r, H}}\left|I_{\mathrm{MPTR}}(e)\right|=\Omega\left(n^{2} r^{4}(n)\right)
\end{array}
$$

Recall that a node in $H_{r}$ is connected to all and only those nodes that are placed in adjacent squarelets. Hence Lemma 4.10 tells us that the degree of each vertex $v \in H_{r}$ is bounded as

$$
4 c_{1} n r^{2}(n) \leq \operatorname{deg}(v) \leq 4 c_{2} n r^{2}(n)
$$

Now lets prove the lower bound by considering the model MPTR. According to Definition 3.6, the transmission on edge $e$ experiences interference from any transmission by a node $v$ such that $r(n)<\left\|X_{e^{-}}-X_{v}\right\| \leq(1+\eta) r(n)$. Therefore, there exists an annular ring around $e^{-}$of width $\eta r(n)$ such that any transmission from a node in this ring interferes with $e$. The area of this annular ring is given by

$$
\text { area of annular ring }=\eta(2+\eta) \pi r^{2}(n)
$$


We have already seen ( Lemma 4.2) that an area of $\Theta\left(r^{2}(n)\right)$ contains at least $\Theta\left(n r^{2}(n)\right)$ nodes. Hence, there exists a $c_{3}$ such that

$$
\gamma_{\text {min }} \geq c_{3} n r^{2}(n) \times 4 c_{1} n r^{2}(n)
$$

Eq. (48) proves the reqd. lower bound. The proof for the upper bound is obtained with a similar argument. First, let us inspect the transmission along edge $e$ under the SPR model. Any transmission from a node in a disk of radius $(1+\eta) r(n)$ around $e^{-}$, interferes with $e$. Moreover, a transmission from $e^{+}$interferes with any reception in a disk of radius $(1+\eta) r(n)$ around $e^{+}$. Given that $\left\|X_{e^{+}}-X_{e^{-}}\right\| \leq r(n)$, there exists a disk of radius $(2+\eta) r(n)$ around $e^{-}$containing all the nodes that may have transmission conflicts with $e$. Further note that a squarelet can be completely inscribed in a circum-circle of radius $(1 / 3 \sqrt{2}) r(n)$. Hence, there exists a circle of maximum radius $R=(2+\eta+(1 / 3 \sqrt{2})) r(n)$ containing all the nodes that may have transmission conflicts with $e$ under the model RSPR. The area of $R$ is $\Theta\left(r^{2}(n)\right)$ and hence there exists a constant $c_{4}$ such that

$$
\begin{aligned}
\gamma_{\max } & =\max _{e \in E_{r, H}}|W(e)| \\
& \leq(\max . \text { no. of nodes in } R) \times(\text { max. node degree }) \\
& \leq c_{4} n(2+\eta+(\sqrt{2} / 6))^{2} r(n)^{2} \times 4 c_{2} n r^{2}(n) \\
& =O\left(n^{2} r^{4}(n)\right)
\end{aligned}
$$

Lemma 5.14: Consider the graph $H_{r}$ with $r(n) \geq r_{c}(n)$ and $k \geq \Theta(n)$. In such a graph, each edge $e$ has at least $\Theta\left(n r^{2}(n)\right)$ clones under interference $I_{\text {RMPR }}$ and $\Theta\left(n^{2} r^{4}(n)\right)$ clones under interference $I_{\text {RMPTR }}$, such that these clones interfere with each other and $e$, under the interference $I_{\mathrm{RSPR}}$.

Proof: According to Definitions 5.10 and 5.11, $U(e)$ and $V(e)$ represent the desired set of clones for $I_{\mathrm{RMPR}}$ and $I_{\mathrm{RMPTR}}$ respectively. Lemma 4.10 implies that there exist $c_{1}$ and $c_{2}$ such that

$$
\begin{aligned}
\mu_{R M P R} & =\min _{e \in E_{r, H}}|U(e)| \\
& \geq \min . \text { vertex degree }=c_{1} n r^{2}(n)
\end{aligned}
$$

$$
\begin{aligned}
\mu_{R M P T R} & =\min _{e \in E_{r, H}}|V(e)| \\
& \geq\left[\min _{e \in E_{r, H}}|U(e)|\right] \times \text { min. nodes per squarelet } \\
& \geq c_{1} n r^{2}(n) \times c_{2} n r^{2}(n)
\end{aligned}
$$

Theorem 5.15: For $r(n) \geq r_{c}(n)$ and $k \geq \Theta(n)$, the capacity of random geometric network is at least: (a) $\Theta(n r(n) / k)$ under the SPR model, (b) $\Theta(n r(n) / k)$ under the MPR model, and (c) $\Theta\left(n^{2} r^{3}(n) / k\right)$ under the MPTR model.

Proof: Recall that the capacity of the random network is greater than the feasible flow rate in $H_{r}$. Theorem 4.12 shows that a rate of $f=c_{1} n^{2} r^{3}(n) / k$ is feasible in $H_{r}$. Additionally, Lemma 5.13 shows that the size of the largest interference set under RSPR is at most $\kappa=c_{2} n^{2} r^{4}(n)$. Hence, Lemma 5.4 implies that a rate of

$$
f_{R S P R}=f \times(1 /(1+\kappa)) \geq\left(c_{3} / r(n) k\right)
$$

is feasible under the RSPR model. If we take into consideration the interference clones, then Lemma 5.6 further implies that the rate

$$
\begin{aligned}
f_{R M P R} & =f \times(1 /(1+\kappa)) \times\left(1+\mu_{R M P R}\right) \\
& \geq\left(c_{3} / r(n) k\right) \times\left(c_{4} n r^{2}(n)\right) \\
& =\left(c_{3} c_{4} n r(n) / k\right)
\end{aligned}
$$

is feasible under the RMPR model. Similarly, the rate

$$
\begin{aligned}
f_{R M P R} & =f \times(1 /(1+\kappa)) \times\left(1+\mu_{R M P T R}\right) \\
& \geq\left(c_{3} / r(n) k\right) \times\left(c_{5} n^{2} r^{4}(n)\right) \\
& =\left(c_{3} c_{5} n^{2} r^{3}(n) / k\right)
\end{aligned}
$$

is feasible under the RMPTR model. Finally, note that a feasible rate under a restricted model is necessarily feasible under the original model. Hence, the result proven in Lemma 5.13 completes the proof.

\section{Upper Bounds}

The interference-free capacity provides an upper bound on the capacity under any model, and Theorem 5.15 already shows that the MPTR model achieves this capacity. However, we need to provide additional arguments to obtain a tight bound on the capacity under SPR and MPR. Our arguments are similar to [8] and [6], and we briefly sketch the proof of the following result for the sake of completeness.

Theorem 5.16: For $r(n) \geq r_{c}(n)$ and $k \geq \Theta(n)$, the capacity of random geometric network is at most: (a) $\Theta(1 / r(n) k)$ under the SPR model, (b) $\Theta(n r(n) / k)$ under the MPR model, and (c) $\Theta\left(n^{2} r^{3}(n) / k\right)$ under the MPTR model.

Proof: (Sketch) Consider the Cut $S$ in Figure 1. The capacity of this cut and hence the network is less than

$$
\frac{(\text { no. of transmitters in } A) \times(\text { max. tranmission per node })}{(1 / 2) k}
$$

The total number of transmitters in $A$ is less than the total number of nodes in $A$. We have already shown that the total number of nodes is $\Theta(n r(n))$. Under the MPTR model, each node can transmit a maximum of $\Theta\left(n r^{2}(n)\right)$ packets, and under the MPR model each packet trasnmits just a single packet. This provides the bounds for MPR and MPTR. To obtain the bound for SPR, we note that each transmitter silences all the nodes within an area of $\Theta\left(r^{2}(n)\right)$. Hence, only $\Theta(1 / r(n))$ nodes are capable of transmitting simultaneously across a cut. Each of these nodes transmits a single packet and, therefore, the above equation provides the bound for SPR.

\section{DISCUSSION}

The results we have presented demonstrate that future ad hoc networks can scale well beyond the Gupta-Kumar capacity bounds attained when nodes simply try to avoid MAI [1]. First, we showed that the optimal capacity that any protocol architecture can attain in a wireless network is $\Theta\left(n^{2} r^{3}(n) / k\right)$. Second, we demonstrated that this capacity can indeed be attained when nodes embrace MAI as transmitters and receivers, and that a non-vanishing capacity is attainable per S-D pair 
even when information must be disseminated over multiple hops. Given that the majority of nodes in an ad hoc network are sources and destinations of information, we frame the rest of our discussion for the important case of $k=\Theta(n)$.

The capacity under the SPR model when $k=\Theta(n)$ equals $\Theta(1 / n r(n))$, which is the well-known Gupta-Kumar result, and shows that avoiding MAI in the communication protocols of ad hoc networks does not scale. Given that today's ad hoc networks are based on interference avoidance, it is clear that massively scalable ad hoc networks cannot exist without substantial changes to the way in which protocols are designed.

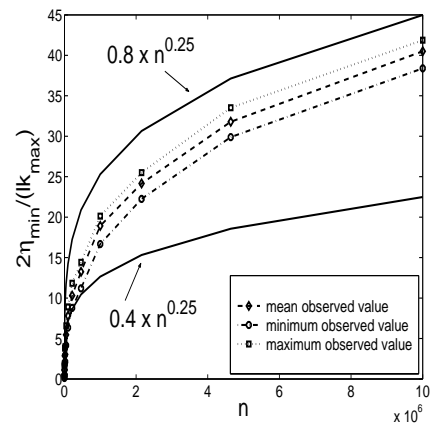

Fig. 4. Simulation result of per-node capacity as a function of $n$

On the other hand, under the MPR model [6] the network capacity is equal to $\Theta(r(n))$, which constitutes a dramatic improvement over SPR, and promises to provide capacity gains in practice by embracing interference at the receivers. However, to attain a non-vanishing capacity, $r(n)$ must be $\Theta(1)$, i.e., use single-hop communication. Unfortunately, this is not feasible in practice, because of the energy that would be incurred in transmissions and the complexity required for the receivers to decode a number of transmissions in the order of the nodes in the network.

In contrast to the above, MPTR achieves the optimal pernode capacity of $\Theta\left(n r^{3}(n)\right)$. Thus, any choice of $r(n)=$ $\Omega\left(n^{1 / 3}\right)$ allows us to increase the per-node capacity of the network with $n$, while still having multihop communication. Moreover, the transmission range and hop-size decreases with $n$. As per our analysis, the capacity of a network is a constant factor of $\Gamma$, where $\Gamma=2 \eta_{\min } / l\left(k_{\max }\right)$, such that $\eta_{\min }$ is the minimum number of edges between any two squarelets, $k_{\max }$ is the maximum number of sources or destinations in a single squarelet and $l \times l$ is the total number of squarelets. To illustrate this, we numerically evaluated the behavior of $\Gamma$ as a function of $n$. Figure 4 presents the mean, minimum and maximum observed value of $\Gamma$ over a thousand network topologies randomly generated and in which $k=n / 2$ and $r(n)=1 / n^{0.25}$. Clearly, as $n \rightarrow \infty$ we have $r(n) \rightarrow 0$. Nevertheless, the numerical results show that the per-node capacity still increases as $\Theta\left(n^{0.25}\right)$.

In closing, we should point out that, while our results provide a completely new outlook on the design of wireless ad hoc networks, much work remains to be done to fully understand their fundamental limits! For example, the results we have presented address only unicast traffic; our model can be used to study the cases of multicast and broadcast information dissemination. We also hope that this paper motivates research on protocol architectures that combine multi-packet reception and transmission to attain massively scalable ad hoc networks.

\section{REFERENCES}

[1] P. Gupta and P. R. Kumar, "The capacity of wireless networks," IEEE Transactions on Information Theory, vol. 46, no. 2, pp. 388-404, 2000.

[2] R. Ahlswede, C. Ning, S.-Y. R. Li, and R. W. Yeung, "Network information flow," IEEE Transactions on Information Theory, vol. 46, no. 4, pp. 1204-1216, 2000.

[3] Y. Wu, P. A. Chou, and S.-Y. Kung, "Information exchange in wireless networks with network coding and physical-layer broadcast," in Proc. of CISS 2005, Baltimore, MD, March 16-18 2005.

[4] S. Katti, S. Gollakota, and D. Katabi, "Embracing wireless interference: Analog network coding," in Proc. of ACM SIGCOMM 2007, Kyoto, Japan, August 27-31 2007.

[5] R. M. de Moraes, H. R. Sadjadpour, and J. J. Garcia-Luna-Aceves, "Many-to-many communication: A new approach for collaboration in manets," in Proc. of IEEE INFOCOM 2007, Anchorage, Alaska, USA., May 6-12 2007.

[6] J. J. Garcia-Luna-Aceves, H. R. Sadjadpour, and Z. Wang, "Challenges: Towards truly scalable ad hoc networks," in Proc. of ACM MobiCom 2007, Montreal, Quebec, Canada, September 9-14 2007.

[7] S. Toumpis and A. J. Goldsmith, "Capacity regions for wireless ad hoc networks," IEEE Transactions on Wireless Communications, vol. 2, no. 4, pp. 736-748, 2003.

[8] J. Liu, D. Goeckel, and D. Towsley, "Bounds on the gain of network coding and broadcasting in wireless networks," in Proc. of IEEE INFOCOM 2007, Anchorage, Alaska, USA., May 6-12 2007.

[9] L. R. Ford and D. R. Fulkerson, Flows in Networks. Princeton Univ. Press, 1962.

[10] T. Leighton and S. Rao, "Multicommodity max-flow min-cut theorems and their use in designing approximation algorithms," Journal of the ACM, vol. 46, no. 6, pp. 787-832, 1999.

[11] P. Klein, S. A. Plotkin, and S. Rao, "Excluded minors, network decomposition, and multicommodity flow," in Proc. of ACM symposium on Theory of computing, San Diego, California, USA, May 16-18 1993.

[12] O. Gunluk, "A new min-cut max-flow ratio for multicommodity flows," SIAM Journal of Discrete Mathematics, vol. 1, no. 1, pp. 1-15, February 2007.

[13] M. Franceschetti, O. Dousse, D. Tse, and P. Thiran, "Closing the gap in the capacity of wireless networks via percolation theory," IEEE Transactions on Information Theory, vol. 53, no. 3, pp. 1009-1018, 2007.

[14] M. Grossglauser and D. Tse, "Mobility increases the capacity of ad hoc wireless networks," IEEE/ACM Transactions on Networking, vol. 10, no. 4, pp. 477-486, 2002.

[15] P. Kyasanur and N. Vaidya, "Capacity of multi-channel wireless networks: Impact of number of channels and interfaces," in Proc. of ACM MobiCom 2005, Cologne, Germany, August 28-September 22005.

[16] A. Ozgur, O. Leveque, and D. Tse, "Hierarchical cooperation achieves optimal capacity scaling in ad hoc networks," IEEE Transactions on Information Theory, vol. 53, no. 10, pp. 2549-3572, 2007.

[17] - "Hierarchical cooperation achieves optimal capacity scaling in ad hoc networks," IEEE Transactions on Information Theory, vol. 53, no. 10, pp. 2549-3572, 2007.

[18] R. Madan, D. Shah, and O. Leveque, "Product multicommodity flow in wireless networks," Submitted to IEEE Transactions on Information Theory, 2007.

[19] M. Kunde, "Block gossiping on grids and tori: Deterministic sorting and routing match the bisection bound," in Proc. of European Symp. Algorithms, 1991.

[20] S. Kulkarni and P. Viswanath, "A deterministic approach to throughput scaling wireless networks," IEEE Transactions on Information Theory, vol. 50, no. 6, pp. 1041-1049, 2004.

[21] B. Bollabas, Modern Graph Theory. Springer Verlag, 1998. 